\title{
Bikinis Instigate Generalized Impatience in Intertemporal Choice
}

\author{
BRAM VAN DEN BERGH \\ SIEGFRIED DEWITTE \\ LUK WARLOP*
}

\begin{abstract}
Neuroscientific studies demonstrate that erotic stimuli activate the reward circuitry processing monetary and drug rewards. Theoretically, a general reward system may give rise to nonspecific effects: exposure to "hot stimuli" from one domain may thus affect decisions in a different domain. We show that exposure to sexy cues leads to more impatience in intertemporal choice between monetary rewards. Highlighting the role of a general reward circuitry, we demonstrate that individuals with a sensitive reward system are more susceptible to the effect of sex cues, that the effect generalizes to nonmonetary rewards, and that satiation attenuates the effect.
\end{abstract}

$\mathrm{A}$ dvertisers search for a way to break through the clutter by using sexually oriented appeals in marketing campaigns. Previous research on the use of sexual imagery in advertising has focused on, among other things, consumer's brand recall and recognition, appeal evaluation, attention, purchase intentions, and product perception. The current study differs from earlier work by showing that the consequences of using sexual imagery extend further than the evaluation of the product or brand itself. In line with previous research demonstrating that exposure to sexual cues influences economic decision making (e.g., Van den Bergh and Dewitte 2006; Wilson and Daly 2004), we will argue that exposure to sexual cues may affect such decisions as

\footnotetext{
*Bram Van den Bergh (Bram.VandenBergh@econ.kuleuven.be) is a doctoral student in marketing, Siegfried Dewitte is associate professor of marketing (Siegfried.Dewitte@econ.kuleuven.be), and Luk Warlop (Luk.Warlop@econ.kuleuven.be) is professor of marketing, all at the KULeuven, Faculty of Business and Economics, Naamsestraat 69, B3000 Leuven, Belgium. The first author is supported by a PhD fellowship of the Research Foundation-Flanders (FWO), and the second and third authors are supported by grant G.0391.03 from the FWO and a research grant of the KULeuven (OT 03/07). Financial support by CensydiamSynovate is gratefully acknowledged. The authors thank the editor, the associate editor, three anonymous reviewers, Simona Botti, Vladas Griskevicius, Gita Johar, Davy Lerouge, Tom Meyvis, participants of the EMAC doctoral colloquium in Reykjavik in May 2007, participants at the ACR conference in Orlando in September 2006, and the consumer behavior group at the KULeuven for their very helpful and valuable comments. Address correspondence to Bram Van den Bergh.
}

John Deighton served as editor and Baba Shiv served as associate editor for this article.

Electronically published January 18, 2008 whether to purchase a less expensive item that can be enjoyed now or to save for a more expensive one.

Choice is relatively easy when choice alternatives differ on a single dimension: individuals generally prefer a larger over a smaller reward, as well as a sooner provided over a later provided reward. Problems arise when choice options differ on more than one dimension. The choice between a smaller reward available sooner and a larger reward available later is less obvious because it involves trading off costs and benefits occurring at different times (Frederick, Loewenstein, and O'Donoghue 2002; Green and Myerson 2004; Soman et al. 2005). Delaying a reward reduces the value of that reward, and this delay discounting process may be influenced by visceral factors (Loewenstein 1996). An increase in desire, by physical or temporal proximity to rewards, may instigate impatience (Baumeister 2002; Hoch and Loewenstein 1991) and steeper delay discounting of rewards. Recent neuroscientific research suggests that factors producing limbic activation, such as the sight, smell, or touch of a desired object, may be associated with impulsive behavior (McClure et al. 2004). Evidence that speaks to this comes from, among others, studies employing the delay-of-gratification paradigm (Metcalfe and Mischel 1999). In a typical experiment, a child is offered a less preferred reward and is confronted with the dilemma to wait 15 minutes and receive a more preferred reward or forgo the more preferred reward and immediately receive just the less preferred reward. When the rewards are out of sight, $75 \%$ of the children wait 15 minutes to obtain the more preferred reward. However, when the rewards are exposed, the mean delay time drops from more than 11 minutes to about 1 minute. None of the children is capable of resisting temptation or delaying gratification when the rewards are within 
reach (Mischel and Ebbesen 1970). The exposed rewards (i.e., "hot stimuli"; Metcalfe and Mischel 1999) increase desire and engender impatience (Baumeister 2002; Hoch and Loewenstein 1991), possibly through activation of the limbic system (McClure et al. 2004). The bulk of prior research has demonstrated specific motivational effects of desire (e.g., an increase in desire for food, by exposure to food rewards, leads to impatience for food rewards). On the contrary, we hypothesize that an increase in appetitive motivation may instigate generalized impatience in intertemporal choice through a common reward processing circuitry. We propose that exposure to "hot stimuli" (Metcalfe and Mischel 1999) leads to a nonspecific time perspective collapse toward the present. Based on recent neurological findings suggesting that rewards are processed similarly in the brain, we propose that exposure to "hot stimuli" may instigate general impatience in intertemporal choice. We will argue that a greater appetite causes a greater urgency to consume anything rewarding.

\section{CONCEPTUAL FRAMEWORK}

Visceral factors may drive impatient and impulsive behaviors (Loewenstein 1996): hungry people order more food than they can eat (the "eyes bigger than your stomach" effect), buy more food than originally intended (Gilbert, Gill, and Wilson 2002; Nisbett and Kanouse 1969), have a stronger preference for candy over fruit (Read and van Leeuwen 1998), and demonstrate less self-control (defined by a greater preference for smaller, less delayed access to apple juice; Kirk and Logue 1997). Other visceral factors such as drug craving cause equivalent short-sighted decisions: heroin addicts have a heightened preference for smaller, sooneravailable amounts of heroin over larger, delayed amounts when they are opioid deprived but not when they are opioid satiated (Giordano et al. 2002). Likewise, nicotine deprivation causes smokers to become more impatient, defined by an increased preference for immediately available cigarettes (Field et al. 2006; Mitchell 2004). Like other visceral factors, sexual desire may lead to impulse control difficulties. After exposure to photographs high in sex appeal (Blanton and Gerrard 1997) or during masturbation (Ariely and Loewenstein 2006), men lower their risk estimates for sexually transmitted diseases. In general, visceral factors, such as hunger, drug craving, or sexual desire, bring about myopic, impulsive, or impatient decisions.

Previous studies on the impact of visceral factors assume that temporal myopia is good specific. The implicit assumption that a hungry person would only make short-sighted trade-offs between immediate and delayed food (and not between immediate and delayed money) is pervasive (Kirk and Logue 1997; Read and van Leeuwen 1998). Loewenstein (1996) explicitly stated that the present orientation applies only to goods that are associated with the visceral factor. Nonetheless, many rewards are processed similarly in the brain (Montague, King-Casas, and Cohen 2006). Neural evidence suggests that the same dopaminergic reward circuitry of the brain is activated for a wide variety of different rein- forcers (Camerer, Loewenstein, and Prelec 2005). That is, a similar set of brain reward regions responds in common to very distinct categories of reward-for example, beautiful female faces and erotic stimuli activate the classical reward circuitry that had already been associated with drug and monetary rewards in prior research (Aharon et al. 2001; Stark et al. 2005). Theoretically, a general neurological system processing rewards may give rise to nonspecific effects (Wadhwa, Shiv, and Nowlis 2006). Visceral factors may thus give rise to generalized temporal myopia. A change in the general time preference may have an impact on all intertemporal choices, including those that are unrelated to the factor that caused the change in one's general time preference. Time preference changes may thus be observed in seemingly unrelated domains. Several empirical studies provide support for such an account. Heroin addicts not only prefer smaller, immediate amounts of heroin over larger, delayed amounts of heroin when they are opioid deprived but also prefer smaller, sooneravailable monetary rewards over larger, delayed monetary rewards when in a drug-craving state (Giordano et al. 2002). Likewise, smokers display more pronounced delay discounting not only of cigarettes after nicotine deprivation but also of monetary rewards (Field et al. 2006). Most relevant to the current research is the finding that delay discounting of monetary rewards increases in men who viewed attractive women relative to men who viewed unattractive women (Wilson and Daly 2004). In a similar vein, we propose that activation of the general reward circuitry, by exposure to "hot stimuli" (i.e., sex cues), leads to general, nonspecific effects in reward processing. We hypothesize that exposure to sex cues causes a nonspecific time perspective collapse toward the present. More specifically, we predict that sexual cues will increase the preference for a smaller and immediate monetary reward over a larger but delayed monetary reward.

\section{H1: Exposure to sex cues will lead to steeper delay discounting of monetary rewards.}

Impulsivity is characterized by generalized reward sensitivity (Ramanathan and Menon 2006). According to Gray's Reinforcement Sensitivity Theory, the Behavioral Approach System (BAS) is the conceptual substrate for sensitivity to rewards (Gray 1987, 1990) and this system responds with appetitive motivation when such cues are encountered. The purpose of the BAS is to initiate approach behavior that brings the organism closer to reinforcers. Sensitivity to rewarding stimuli can vary substantially from one individual to the next (Carver and White 1994; Torrubia et al. 2001). Individuals with a highly sensitive BAS exhibit a greater tendency to respond to rewards with an increased motivation to engage in reward-seeking behaviors and a greater tendency to act on the hedonic impact of reinforcers. Since erotic stimuli activate the human reward circuitry (Stark et al. 2005) and because sensitivity to rewarding stimuli can vary from one individual to the next (Carver and White 1994; Torrubia et al. 2001), we hypothesize that the extent to which one is sensitive to rewards moderates the effects of sexual cues on delay discounting of monetary rewards. 
General temporal myopia should be observed only when the reward system is sensitive enough to be activated by sex cues. We hypothesize that men with a highly reactive or sensitive behavioral approach system should be affected to a greater extent by sexual cues.

H2: Exposure to sex cues will lead to steeper delay discounting of monetary rewards among highsensitive BAS men than among low-sensitive BAS men.

The response to rewards is dependent on the deficit state of an organism. If you do not have money, you may need a smaller sum right now. If you have money, you can afford to wait for a larger sum. Wealthy individuals may display more patience when choosing between a smaller, immediate monetary reward and a larger, more delayed reward than poor individuals. We propose that satiation impedes a nonspecific time perspective collapse toward the present. Indeed, satiation leads to an enhanced capacity of delaying gratification, that is, more patience (Dholakia, Gopinath, and Bagozzi 2005; Giordano et al. 2002; Kirk and Logue 1997). For example, after ad lib smoking, smokers choose significantly more often a larger, delayed reward over a smaller, more immediate reward than after nicotine abstinence (Field et al. 2006). Also, priming studies indicate that goals dissipate in strength once they are satisfied: construct accessibility from motivational sources persists as long as the motivation is active, but accessibility is inhibited upon goal fulfillment (Förster, Liberman, and Higgins 2005). Hence, satiating the induced motivational state should dampen the appetitive response after exposure to "hot stimuli" (Wadhwa et al. 2006). Furthermore, satiation in any domain could attenuate the effect of sex cues on impatience. Indeed, Briers et al. (2006) demonstrated that monetary deprivation (satiation) leads to more (less) food intake. Following the logic of the general reward system, it should come as no surprise that monetary satisfaction is capable of satisfying desires evoked by food. Deprivation in one domain can thus affect appetitive responses in a different domain (Briers et al. 2006). Highlighting the role of a general reward mechanism, we propose that satiation in the monetary domain could attenuate the effect of sex cues on intertemporal choice between nonmonetary rewards. Because low-sensitive BAS individuals are unaffected by sex cue exposure (i.e., hypothesis 2), the effect of satiation should be restricted to high-sensitive BAS individuals.

H3: Compared to low-sensitive BAS men, deprived high-sensitive BAS men will discount any reward more steeply than satiated high-sensitive BAS men after sex cue exposure.

In the following, we will discuss four experimental studies to test these hypotheses. In the first two experiments (studies $1 \mathrm{~A}$ and 1B), we will focus on the main effect of sexual cues on monetary delay discounting (hypothesis 1 ). In studies 2 and 3 , we devote our attention to the moderating effects of
BAS sensitivity (hypothesis 2 and hypothesis 3) and satiation/deprivation (hypothesis 3 ) on delay discounting of rewards across domains.

\section{PREFACE}

In all of our experiments we used a screening procedure to probe attention and motivation. Participants had to answer questions to exclude random response behavior. In studies $1 \mathrm{~A}$ and $1 \mathrm{~B}$, participants had to answer four trivial multiplechoice questions about the rules of an unrelated game; in studies 2 and 3, they were not instructed to respond a scale but instead were asked to click a blue dot next to the question (Oppenheimer, Meyvis, and Davidenko 2007). Data from participants not answering these questions correctly (study 1A: $n=2,7 \%$; study 1B: $n=10,13 \%$; study $2: n=4$, $3 \%$; study $3: n=7,5 \%$ ) were discarded because the responses on focal variables could not be trusted.

\section{STUDY 1A}

In the first study, our aim is to demonstrate that exposure to sexual cues leads to monetary craving. Delaying a reward reduces the value of that reward (Frederick et al. 2002; Green and Myerson 2004), and we hypothesize that proximity to "hot stimuli" leads to an even steeper reduction of subjective value of monetary rewards throughout the time (hypothesis 1).

\section{Participants}

Participants were 42 heterosexual male students at the KULeuven, ranging in age from 18 to 28 years $(M=22$; $\mathrm{SD}=1.98)$. Three students participated in order to receive partial course credit, and 39 students participated in return for a participation fee.

\section{Method}

Participants were seated in partially enclosed cubicles, which prevented them from having contact with each other. In the picture rating task, 15 advertisements were displayed in a random order on a computer screen. Participants rated the attractiveness of the advertisements. Two sets of 15 pictures constituted the stimulus materials. In the "landscape condition," 15 pictures of landscapes (e.g., beaches, fields, mountain ranges, and riversides) were displayed, and in the "sexy women condition," 15 pictures of nonnude female models were shown to the participants. The models assumed different poses and wore diverse outfits, but all were dressed in a sexually appealing manner (e.g., in swimsuit or lingerie). The pictures of the landscapes and sexy women would supposedly be used for a marketing campaign. Participants were instructed to rate the attractiveness of the ads on a seven-point scale ranging from -3 (not attractive at all) to +3 (very attractive).

Subsequently, participants engaged in a delay discounting task. Participants specified the amount of money they would 
require in 1 week and the amount they would require in 1 month to make them indifferent to receiving $€ 15$ now (Thaler 1981). This procedure allows us to specify a discounting function for each participant. Following Myerson, Green, and Warusawitharana (2001), we consider the area under the empirical discounting function as a measure of delay discounting. The area under the curve can vary between 0.0 (steepest possible discounting) and 1.0 (no discounting). (See Myerson et al. [2001] for details regarding the calculation of the area under the curve.) The area measure provides a single and easy statistic that can be used to compare groups and does not depend on any theoretical assumptions regarding the form of the discounting function (Myerson et al. 2001). We employed a hypothetical delay discounting task. Although the use of real rewards is desirable for obvious reasons, there is, as of yet, no clear evidence that hypothetical rewards are discounted differently from real rewards (Johnson and Bickel 2002; Lagorio and Madden 2005; Madden et al. 2003, 2004).

If exposure to sexual cues leads to changes in affect, this may influence impulsivity. For instance, one's mood could influence impulse buying (Rook and Gardner 1993). On the one hand, people who feel happy may be inclined to reward themselves generously and to feel as if they have more freedom to act. On the other hand, when people are upset, they indulge in immediate impulses to make themselves feel better (Tice, Bratslavsky, and Baumeister 2001). Also, emotions of happiness and sadness may influence the likelihood of self-gifts and explain immediate gratification (Mick and Faure 1998). To test whether self-reported mood states were not different across conditions, participants' mood was assessed on a five-point scale ranging from 1 (not at all) to 5 (extremely) by means of the Positive Affect Negative Affect Schedule (PANAS; Watson, Clark, and Tellegen 1988). In addition, we assessed arousal and mood valence by the SelfAssessment Manikin (SAM; Morris 1995), a pictorial scale with five graphic figures. Participants were instructed to indicate which of the five SAM figures corresponds to their current emotional state on a visual analogue scale (100 points). For the arousal dimension, the SAM figures range from a wide-eyed, excited figure to a relaxed, sluggish, or sleepy figure. For the mood valence dimension, SAM ranges from a smiling, happy figure to a frowning, unhappy figure. Positive affect, negative affect, mood valence, and arousal were assessed after the discounting measure to demonstrate that the effect might occur when mood/arousal is not salient. In studies 2 and 3, mood/arousal was assessed prior to the discounting measure.

\section{Results}

Two outliers were removed. An observation is declared an outlier if it lies outside of the interval $[Q 1-1.5 \times$ $\mathrm{IQR} ; Q 3+1.5 \times \mathrm{IQR}$, where $\mathrm{IQR}=Q 3-Q 1$ is called the interquartile range (Tukey 1977). We will use this definition across the four experimental studies. An analysis of variance revealed a significant effect of the content of the advertisements $(F(1,38)=8.54, p<.01)$ on delay discount- ing of money. After exposure to pictures of sexy women, men discounted money more steeply, which was reflected by a smaller area under the empirical discounting function in the sexy women condition $(M=.82, \mathrm{SD}=.11)$ than in the landscape condition $(M=.90, \mathrm{SD}=.06)$; see figure 1 .

The effect of sexual cues on delay discounting is not likely to be driven by mood differences: levels of positive and negative affect (PANAS; Watson, Clark, and Tellegen 1988) did not differ between conditions, and neither did mood valence and arousal (SAM; Morris 1995). Adjusting for positive and negative affect, mood valence, and arousal as covariates did not change the pattern of results reported above, suggesting that mood did not mediate the effect of sexual cues on delay discounting.

Also, the effect of sexual cues on delay discounting cannot be explained by the attractiveness of the pictures. Pictures were rated equally attractive in both conditions (landscape: $M=1.35 ; \mathrm{SD}=.76$; sexy women: $M=0.99 ; \mathrm{SD}=$ $.96 ; F(1,38)=1.71$, NS). Adjusting for the attractiveness of the pictures as covariates did not change the pattern of results, suggesting that attractiveness did not mediate the effect of sexual cues on delay discounting.

\section{FIGURE 1}

\section{DELAY DISCOUNTING OF A MONETARY REWARD (STUDY 1A)}

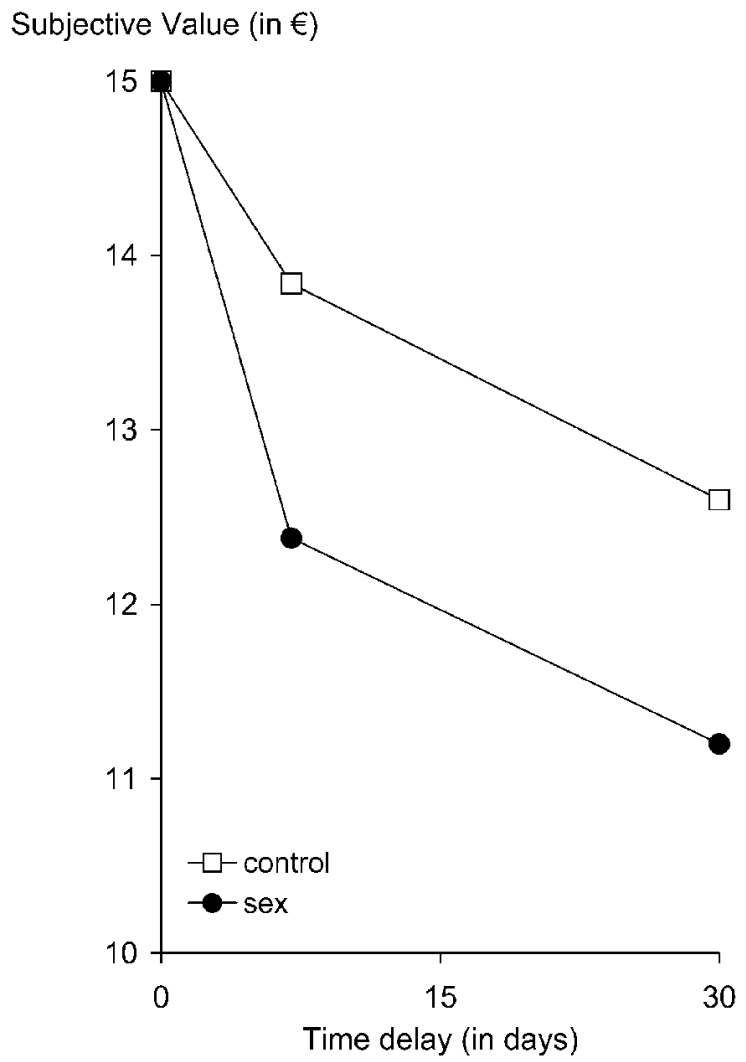




\section{Discussion}

After exposure to sexual cues, men have a heightened preference for immediately available rewards over larger and delayed monetary rewards. Although this is consistent with our contention that sexual cues instigate monetary craving, the mere presence of other persons is likely to influence impulse buying (Luo 2005). It is possible that the mere suggestion of the presence of an individual (in the sexy women condition) versus the absence (in the landscape condition) is causing impatience.

\section{STUDY 1B}

In study 1B our goal is twofold: first, we aim to corroborate the result of study $1 \mathrm{~A}$ that exposure to sexual cues increases impatience in the monetary domain (hypothesis 1); second, we attempt to rule out the possibility that the induced impatience is driven merely by the presence of an individual by using person-free sexual cues. Also, this rules out an explanation in terms of the attractiveness of female models (Wilson and Daly 2004).

\section{Participants}

Participants were 67 heterosexual male students at the KULeuven, ranging in age from 17 to 34 years $(M=21$; $\mathrm{SD}=2.62$ ). Seventeen students participated in order to receive partial course credit, and 50 students participated in return for a participation fee.

\section{Method}

The laboratory setting from the previous study was used. Supposedly to explore the underlying causes of the popularity of several clothing items, participants were asked to rate a piece of clothing on several dimensions. In the "Tshirt condition," a T-shirt was evaluated on several aspects (such as quality and color), and in the "bra condition," a bra was rated on the same dimensions. Eight different Tshirts and eight different bras were randomly allocated to the participants, and participants could evaluate one clothing item by touching, feeling, visually inspecting, and so forth.

Subsequently, participants engaged in a delay discounting task. Participants specified the amount of money they would require in 1 week and the amount they would require in 1 month to make them indifferent to receiving $€ 15$ now. We consider the area under the empirical discounting function as a measure of delay discounting (Myerson et al. 2001). After the delay discounting task, participants' mood was assessed by means of the PANAS (Watson et al. 1988) and arousal and mood valence by the SAM (Morris 1995) to test whether self-reported mood states were not different across conditions.
FIGURE 2

DELAY DISCOUNTING OF A MONETARY REWARD (STUDY 1B)

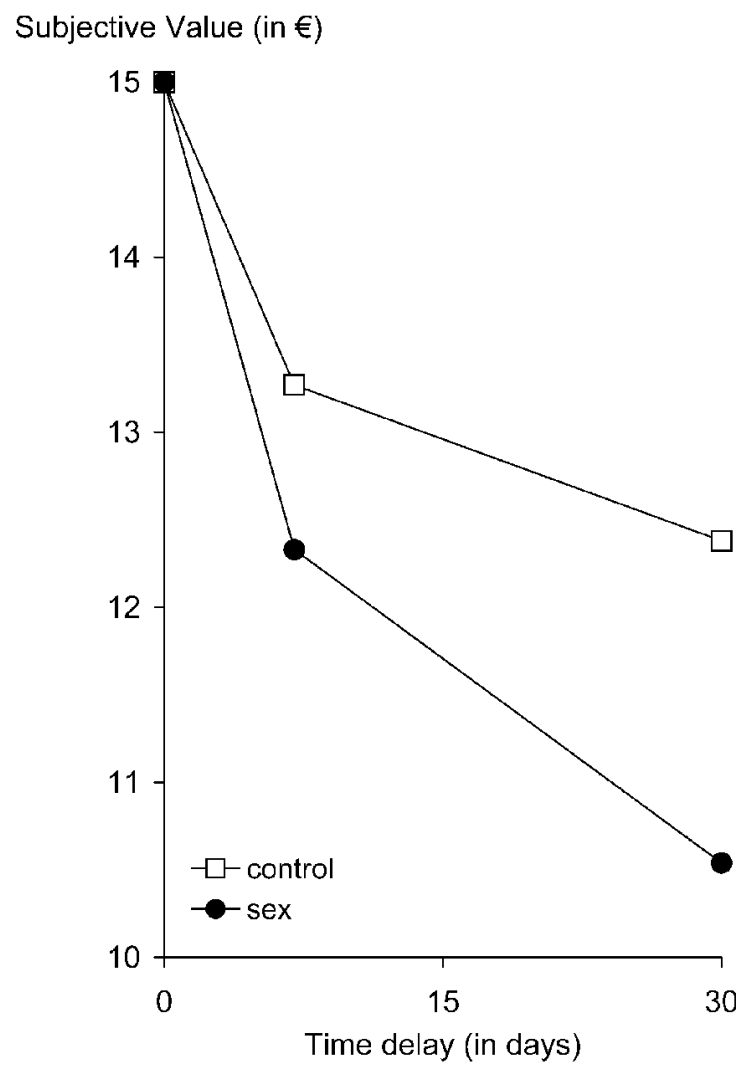

Results

One outlier was removed. An analysis of variance revealed a significant effect of the clothing $(F(1,64)=8.61, p<$ $.005)$ on delay discounting of monetary rewards. After exposure to lingerie, men discounted money more steeply, which was reflected by a smaller area under the empirical discounting function in the bra condition $(M=.80, \mathrm{SD}=.13)$ than in the T-shirt condition $(M=.88, \mathrm{SD}=.08)$; see figure 2 .

The effect of sexual cues on delay discounting is not likely to be driven by mood differences. Levels of positive and negative affect (PANAS; Watson, Clark, and Tellegen 1988) did not differ between conditions, and neither did mood valence and arousal (SAM; Morris 1995). Adjusting for positive and negative affect, mood valence, and arousal as covariates did not change the pattern of results reported above, suggesting that mood did not mediate the effect of sexual cues on delay discounting.

\section{Discussion}

Male participants require more money in the future to make them indifferent to receiving $€ 15$ now after exposure to a bra than after exposure to a T-shirt. That is, sexual cues 
lead to steeper discounting of money. This finding is consistent with study $1 \mathrm{~A}$ and suggests that sexual cues lead to a nonspecific collapse of time perspective toward the present, supporting the role of a general reward system. Unlike earlier research, steeper discounting of monetary rewards after exposure to sexual cues cannot be due to the attractiveness of female models (Wilson and Daly 2004) and is not driven merely by the presence of an individual (Luo 2005). In studies 2 and 3, we explore the underlying mechanism giving rise to this effect. More specifically, we will focus on the moderating effects of BAS sensitivity and satiation/deprivation.

\section{STUDY 2}

Our goal in the second study is twofold. First, our aim is to demonstrate that men with a highly sensitive reward system are more likely to be influenced by sexual cues (hypothesis 2). Our second objective is ruling out an explanation in terms of distraction or cognitive capacity.

The BAS is sensitive to reward stimuli and responds with appetitive motivation when rewards are encountered. Since erotic stimuli activate the human reward circuitry (Stark et al. 2005) and because sensitivity to rewarding stimuli can vary from one individual to the next (Carver and White 1994; Torrubia et al. 2001), we conjecture that the extent to which one is sensitive to rewards moderates the effects of sexual cues on delay discounting of monetary rewards. A time preference shift should be observed only when the reward system is sensitive enough to be activated by sex cue exposure. More specifically, we hypothesize that men with a highly sensitive behavioral approach system (as assessed with the Sensitivity to Punishment/Sensitivity to Reward Questionnaire [SPSRQ]; Torrubia et al. 2001) discount monetary rewards more steeply after sex cue exposure than men who are insensitive to rewards.

To the extent that sexual cues distract or cognitively load participants, the reported effects in our earlier studies could be due to lower working memory capacity (Shiv and Fedorikhin 1999). Indeed, Hinson, Jameson, and Whitney (2003) found that a secondary task (a digit memory task) increased the preference for immediate rewards in a delay discounting task. It is well known that cognitive load decreases performance on a variety of cognitive tasks (Baddeley 1996; Baddeley, Chincotta, and Adlam 2001; Toms, Morris, and Ward 1993). If sexual cues increase working memory load, performance on cognitive tasks should decrease. The Remote Associates Test (RAT; Mednick 1962) is a cognitive test measuring creativity, whereby creativity is narrowly defined as the ability to make rapid appropriate associations between various concepts. A RAT question consists of providing people with three words (e.g., "dress, dial, and flower") and giving them a limited amount of time (15 seconds in the current study) to come up with the one correct word linked to all three of the original words ("sun"). If sex cues would impair working memory capacity, a decrease in performance should be observed in the sex cue condition. In contrast, we hypothesize that an amelioration of RAT performance will be observed after exposure to a sex cue (Griskevicius, Cialdini, and Kenrick 2006). Because activation of the reward circuitry increases creativity (Eisenberger, Armeli, and Pretz 1998), our theory explicitly predicts that RAT performance should vary in an analogous way as delay discounting. That is, the increase in RAT performance after sex cue exposure should be more pronounced among men who possess a sensitive reward system.

\section{Participants}

Participants were 120 heterosexual male students at the KULeuven, ranging in age from 18 to 39 years $(M=21$; $\mathrm{SD}=2.31$ ). All students participated in return for a participation fee.

\section{Method}

The laboratory setting from the previous studies was used. All participants first viewed a 1-minute advertising commercial. In the "control condition," the commercial video featured hundreds of men sprinting through scenery, and in the "sex cue condition," the commercial video contained hundreds of young women, dressed in bikinis, running across hills, fields, and beaches. Participants were instructed to watch the commercial carefully since they would be asked questions about it at a later point in time.

To test whether self-reported mood states were not different depending on condition, participants' mood was assessed immediately after exposure to the commercial by means of the PANAS (Watson et al. 1988), mood valence, and arousal on a visual analogue scale by the SAM (Morris 1995).

Subsequently, participants engaged in a delay discounting task. Participants specified the amount of money they would require in 1 week and the amount they would require in 1 month to make them indifferent to receiving $€ 15$ now. As in studies $1 \mathrm{~A}$ and $1 \mathrm{~B}$, we consider the area under the empirical discounting function as a measure of delay discounting (Myerson et al. 2001).

To rule out a distraction explanation, the RAT was administered. Participants had to solve five RAT questions and had 15 seconds to answer each question. The correct responses to the RAT questions were summed and subjected to statistical analyses.

Afterward, respondents answered the SPSRQ (Torrubia et al. 2001), a scale specially developed to assess Gray's behavioral approach and inhibition constructs that consists of 48 yes/no items such as "Do you often do things to be praised?" (SR) and "Are you often afraid of new or unexpected situations?" (SP). The 24 Sensitivity to Reward (SR) items were averaged to obtain a SR score (Cronbach's $\alpha=.71)$. Including the Sensitivity to Punishment scale in statistical analyses produced no significant effects and is therefore ignored in the remainder of the study. 


\section{Results}

For the analysis on the delay discounting task, four outliers were removed. A general linear model (GLM) analysis was used for the analysis. The GLM combines features of ANOVA and regression-based models and can therefore handle any combination of continuous and discrete variables. In the analysis, Commercial (sexy/control) was entered as a discrete between-subjects factor, whereas Sensitivity to Reward (SR) was entered as a continuous between-subjects factor. This GLM revealed a significant effect of the content of the commercial on delay discounting of money $(F(1,112)=5.14 ; p<.05)$. After exposure to a sexy commercial, men discounted money more steeply, which was reflected by a smaller area under the empirical discounting function in the sexy cue condition $(M=.84$, $\mathrm{SD}=.12)$ than in the control condition $(M=.85, \mathrm{SD}=$ .11). Furthermore, the analysis yielded a significant main effect of SR scores $(F(1,112)=11.43$; $p<.005)$, demonstrating that men with a more sensitive reward system discounted money more steeply than men with a less sensitive appetitive system $(r=-.29, p<.005, n=116)$. These two main effects were qualified by a significant interaction between the content of the commercial and SR scores $(F(1,112)=6.40 ; p<.05)$. That is, the correlation between SR scores and discounting was not significant in the control condition ( $r=-.08, p=.54, n=58$ ), but there was a significant negative correlation between SR scores and discounting in the sex cue condition $(r=-.48, p<.0001$, $n=58$ ), indicating that greater sensitivity for reward is associated with a smaller area under the empirical delay discounting function of money, but only when men have been previously exposed to a sexy commercial. To visualize these data, the distribution of the SR scores was dichotomized on the basis of a median split to define high- and low-sensitive-to-reward groups $\left(\mathrm{Mdn}=14 ; \quad M_{\text {high }}=\right.$ $\left.16.89, M_{\text {low }}=10.67\right)$. Planned contrasts revealed that the high-sensitive-to-reward group discounts rewards more steeply $(t(112)=1.96, p=.05)$ after exposure to a sexy commercial $(M=.77, \mathrm{SD}=0.13)$ than after exposure to a control commercial $(M=.83, \mathrm{SD}=.12)$; see figure 3 . Within the low-sensitive-to-reward group however, exposure to sex cues did not lead to steeper discounting of rewards $(t(112)=0.70, \mathrm{NS})$. That is, after sex cue exposure, men with a sensitive reward system discount rewards more steeply $(t(112)=4.31, p<.0001)$ than men who are insensitive to rewards $(M=0.88, \mathrm{SD}=.07)$; see figure 3 .

A GLM analysis revealed a marginally significant effect of the content of the commercial on RAT performance $(F(1,116)=3.16 ; p=.078)$. After exposure to a sexy commercial, men were more likely $(M=2.05, \mathrm{SD}=$ 1.34) to come up with the one correct word (e.g., "sun") linked to all three of the original words (e.g., "dress, dial, and flower") than in the control condition $(M=1.92$, $\mathrm{SD}=1.36)$. The analysis yielded no significant main effect of SR scores $(F(1,116)=1.29 ; p=.26)$, but a significant interaction between condition and SR scores was obtained $(F(1,116)=4.05 ; p<.05)$. That is, the correlation between
FIGURE 3

DELAY DISCOUNTING OF A MONETARY REWARD (STUDY 2)

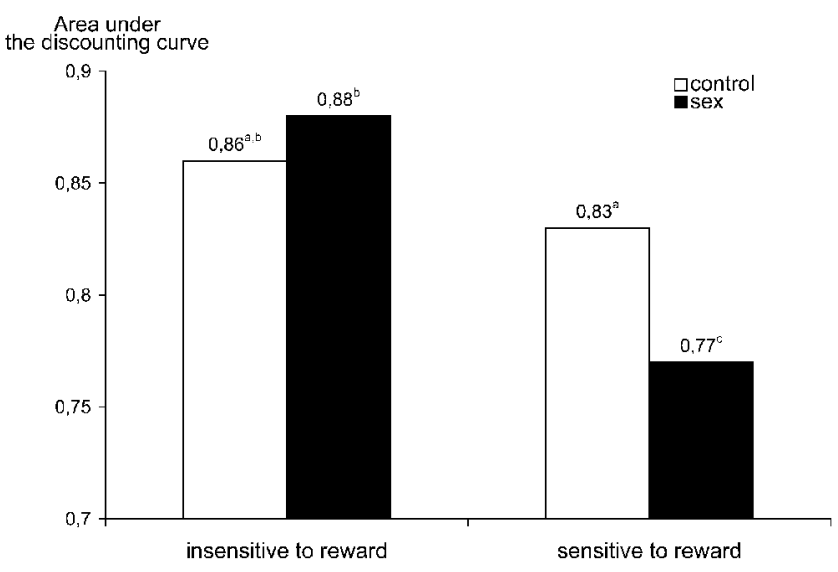

NOTE.-Different superscripts indicate that the difference between means is significant $(p \leq .05)$.

SR scores and RAT performance was not significant in the control condition $(r=-.08, p=.53, n=60)$, but there was a significant positive correlation between SR scores and RAT performance in the sex cue condition $(r=.28, p<$ $.05, n=60)$, indicating that greater sensitivity for reward is associated with better performance on a cognitive task but only after exposure to a sexy commercial. Planned contrasts revealed that the high-sensitive-to-reward group $(M=2.5, \mathrm{SD}=1.37)$ is more likely to come up with one correct word linked to all three of the original words after a sexy commercial $(t(116)=2.17, p<.05)$ than the lowsensitive-to-reward group $(M=1.75, \mathrm{SD}=1.22)$. After the control commercial, however, no differences in RAT performance between the high-sensitive-to-reward group $(M=1.88, \mathrm{SD}=1.18)$ and the low-sensitive-to-reward group $(M=1.97, \mathrm{SD}=1.51)$ was obtained $(t(116)=$ $0.25, \mathrm{NS})$; see figure 4 .

The effect of sexual cues on delay discounting or creativity is not likely to be driven by mood differences. Levels of negative affect, mood valence, and arousal did not differ significantly between conditions. Levels of positive affect (control: $M=3.35, \mathrm{SD}=.61 ;$ sex cue: $M=3.14$, $\mathrm{SD}=.64 ; F(1,118)=3.37, p=.069)$ showed a trend toward significant differences between conditions, but adjusting for positive and negative affect, mood valence, and arousal as covariates did not change the pattern of results reported above, suggesting that mood does not mediate the effect of sexual cues on delay discounting or creativity.

\section{Discussion}

In this second study, we demonstrated that the extent to which one is sensitive to rewards moderates the effects of sexual cues on delay discounting: men with high-sensitive BAS discount monetary rewards more steeply after sex cue 


\section{FIGURE 4}

PERFORMANCE ON REMOTE ASSOCIATES TEST (STUDY 2)

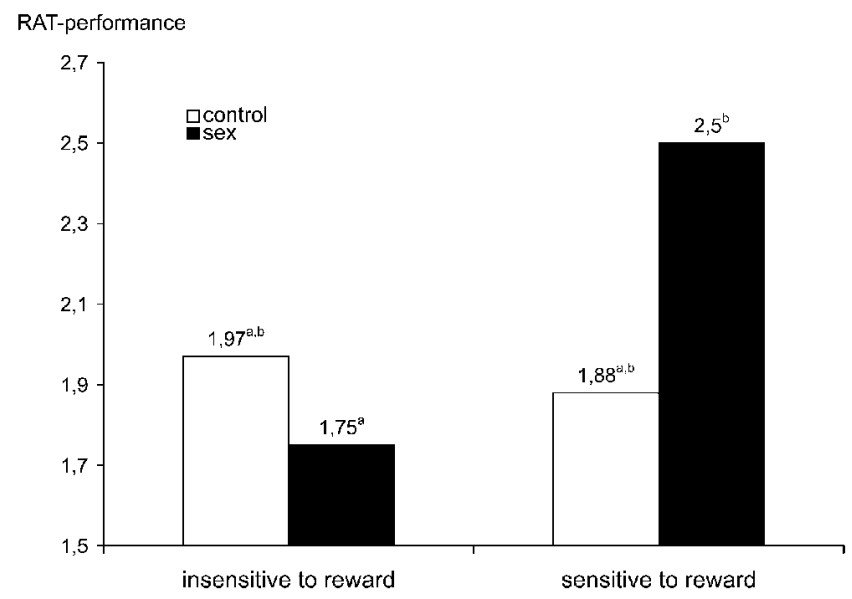

NOTE.-Different superscripts indicate that the difference between means is significant $(p \leq .05)$.

exposure than men who are insensitive to rewards. Hence, activating the reward system by exposure to sexual cues leads to a greater valuation of immediately available monetary resources. Additionally, this experiment rules out an explanation in terms of distraction or working memory load (Hinson et al. 2003; Shiv and Fedorikhin 1999). Consistent with Eisenberger et al. (1998) and Griskevicius et al. (2006), reward-sensitive men perform better on a cognitive creativity task after watching a sexy commercial.

\section{STUDY 3}

In study 3, our goal is threefold. First, we aim to replicate the result of study 2 by demonstrating that the effect of sexual cues on delay discounting is moderated by BAS sensitivity (hypothesis 2; Carver and White 1994; Torrubia et al. 2001). Second, we want to provide further support for the role of a general reward mechanism in delay discounting of rewards by demonstrating that the effect of sexual cues is not restricted to the monetary domain but generalizes to different types of reward, such as candy and soft drinks (hypothesis 3). Additionally, this would rule out an explanation in terms of mate attraction: Roney (2003) demonstrated that exposure to potential mates primes a psychological orientation in men that accords elevated importance to current material wealth. If we could demonstrate effects among rewards unrelated to material wealth, an explanation in terms of mate attraction would be convincingly ruled out. Third, we propose that satiety leads to an enhanced capacity to delay gratification (Dholakia et al. 2005; Field et al. 2006; Giordano et al. 2002; Kirk and Logue 1997): satiating the induced motivational state should attenuate the appetitive response after exposure to "hot stimuli." Since deprivation in one domain can affect appetitive responses in a different domain (Briers et al. 2006), we propose that exposure to sexual cues will lead to steeper delay discounting of such rewards as candy and soft drinks but only when individuals feel monetarily deprived, not when individuals feel monetarily satiated (hypothesis 3). This would provide strong support for our motivational account (i.e., sex cues instigate appetitive motivation) and rule out an explanation in terms of trait priming or behavioral effects of stereotype activation (i.e., sex cues prime the male stereotype and activate the trait of impulsivity; Wheeler and Petty 2001).

\section{Participants}

Participants were 129 heterosexual male students at the KULeuven, ranging in age from 17 to 25 years $(M=19$; $\mathrm{SD}=1.41)$. All students participated in return for course credit.

\section{Method}

The laboratory setting from the previous studies was used. As in study $1 \mathrm{~B}$, participants were asked to rate either a $\mathrm{T}$ shirt ("control condition") or a bra ("sex cue condition") on several dimensions. Eight different T-shirts and eight different bras were randomly allocated to the participants, who could evaluate one clothing item by touching, feeling, visually inspecting, and so forth.

Afterwards, participants were asked to indicate the combined amount of money in their checking and savings accounts. The response scale constituted the independent variable (Nelson and Morrison 2005). Half of the participants were given a nine-point scale divided in €50 increments, from 1 (€0-€50) to 9 (over $€ 400$ ), whereas the other half were given a similar nine-point scale divided in much larger increments, from 1 (€0-€500) to 9 (over $€ 400,000$ ). When participants respond toward the top or bottom of a scale, they tend to make corresponding inferences about their personal circumstances (Schwarz 1999). People responding to the $€ 400,000$ scale feel monetarily deprived, whereas people responding to the $€ 400$ scale feel monetarily satiated (Nelson and Morrison 2005).

To test whether self-reported mood states were not different depending on condition, participants' mood was assessed immediately after the manipulations by means of one item ("How do you feel at this moment?" on a nine-point scale anchored on "very bad" (1) and "very good" (9)) and arousal and mood valence on a visual analogue scale by the SAM (Morris 1995).

Subsequently, participants engaged in a delay discounting task. Participants had to specify how many [euros/cans of soda pop/candy bars] they would require in 1 [week/month] to make them indifferent to receiving 15 [euros/cans of soda pop/candy bars] now (Estle et al. 2007). As in the previous studies, we consider the area under the empirical discounting function of each reward as a measure of delay discounting (Myerson et al. 2001).

Next, BAS sensitivity was assessed. Because of time constraints, we preferred the shorter BIS/BAS scales (20 items; 
Carver and White 1994) over the longer SPSRQ scale (48 items; Torrubia et al. 2001). The BIS/BAS scales yield scores on three BAS subscales (Reward Responsiveness, Fun-seeking, and Drive) and one BIS subscale. For the purposes of this study, only the Reward Responsiveness subscale is utilized because this scale focuses on the responses to the occurrence of reward (Carver and White 1994) and is most relevant for the effect of sexual cues on activation of the general reward system. Including the BIS scale or the other BAS subscales (i.e., Fun-seeking, Drive) in further statistical analyses produced no significant effects, and this is ignored in the remainder of the discussion. Participants responded to nine-point scale items anchored on "strongly disagree" (1) and "strongly agree" (9) on such statements as "When I'm doing well at something, I love to keep at it." The responses to the BAS Reward Responsiveness subscale were averaged to form a composite reward sensitivity score $(\alpha=.71)$.

\section{Results}

Nine outliers were removed. For each reward (money, soda, and candy), the area under the discounting curve was standardized, and these three standardized measures were subjected to a repeated measurement GLM analysis with Motivation (deprivation/satiation), Clothing (bra/T-shirt), Reward Responsiveness (as a continuous factor), and all interactions as independent variables. This analysis revealed no significant within-subjects effects, suggesting that all three discounting measures are affected in a similar way by the independent variables and their interactions (all $F$ 's $<$ 1.30). The between-subject analysis of variance yielded a significant two-way interaction between Motivation and Clothing $(F(1,112)=5.57 ; p<.05)$ and a significant threeway interaction between Motivation, Clothing, and Reward Responsiveness $(F(1,112)=5.81 ; p<.05)$. To explore these interaction effects and to provide an explicit comparison with study 2, two separate GLMs were conducted within the deprivation and satiation conditions. Because study 2 can be considered as a nonsatiation study, we should replicate the effects of study 2 within the deprivation condition of study 3 . Indeed, within the deprivation conditions, a GLM with Reward Responsiveness and Clothing as independent variables yielded a significant main effect of Clothing $(F(1,58)=5.48 ; p<.05)$, a marginally significant effect of Reward Responsiveness $(F(1,58)=3.77 ; p=$ $.06)$, and a significant two-way interaction $(F(1,58)=$ $6.57 ; p<.05)$. Within the satiation conditions, however, neither the main effect nor the interaction effect was significant (all $F$ 's $<1$ ). The significant three-way interaction between Motivation, Clothing, and Reward Responsiveness can be explored by investigating the correlation between the delay discounting measures and Reward Responsiveness within each experimental condition (see table 1).

The correlation between Reward Responsiveness and delay discounting is significant after sex cue exposure but only if individuals feel monetarily deprived $(r=-.46, p<$ $.01, n=32$ ). That is, the more sensitive the reward system,
TABLE1

CORRELATIONS BETWEEN REWARD RESPONSIVENESS AND DELAY DISCOUNTING OF DIFFERENT REWARD ITEMS WITHIN EACH EXPERIMENTAL CONDITION: STUDY 3

\begin{tabular}{llllll}
\hline \hline & \multicolumn{2}{c}{ Sex } & & \multicolumn{2}{c}{ Control } \\
\cline { 2 - 3 } \cline { 5 - 6 } & $\begin{array}{c}\text { Deprivation } \\
(n=32)\end{array}$ & $\begin{array}{c}\text { Satiation } \\
(n=30)\end{array}$ & & $\begin{array}{c}\text { Deprivation } \\
(n=30)\end{array}$ & $\begin{array}{c}\text { Satiation } \\
(n=28)\end{array}$ \\
\hline Money & $-.45^{*}$ & -.17 & & .02 & -.26 \\
Candy & $-.35^{*}$ & .21 & & .17 & -.03 \\
Soda & $-.38^{*}$ & .16 & & .05 & .04 \\
Mean reward & $-.46^{* *}$ & .1 & & .11 & -.09 \\
\hline${ }^{*} p<.05$. & & & & & \\
${ }^{* \star} p<.01$. & & & & & \\
& & & &
\end{tabular}

the steeper the delay discounting curve after exposure to sex cues. This correlation disappears if individuals feel monetarily satiated after sex cue exposure $(r=.10, p=.59$, $n=30$ ). In the T-shirt conditions, no association between discounting and Reward Responsiveness is obtained. As suggested by the absence of within-subjects effects, this correlation pattern is found for each reward separately. That is, touching a bra leads to steeper delay discounting of money, candy bars, and cans of soda pop among men with a sensitive reward system than among men with a less sensitive reward system-but not after monetary satiation (see table 1).

To visualize these data, the distribution of the Reward Responsiveness scores was dichotomized on the basis of a median split to define a high- and low- sensitive-to-reward group, $\left(\operatorname{Mdn}=7.1 ; M_{\text {high }}=7.78, M_{\text {low }}=6.41\right)$. Also, a general delay discounting of rewards index was constructed by averaging the three standardized area-under-the curve measures $(\alpha=0.70)$. Planned contrasts revealed that the high-sensitive-to-reward group, when monetarily deprived, discounts rewards more steeply $(t(119)=3.42, p<.001)$ after exposure to lingerie $(M=-0.47, \mathrm{SD}=1.11)$ than after exposure to a T-shirt $(M=.31, \mathrm{SD}=.45)$; see figure 5 . The high-sensitive-to-reward group, when monetarily satiated, however, did not discount rewards more steeply $(t(119)=1.10, \mathrm{NS})$ after exposure to lingerie $(M=.07$, $\mathrm{SD}=.57)$ than after exposure to a T-shirt $(M=.35$, $\mathrm{SD}=.47)$. That is, after sex cue exposure, men with a sensitive reward system discount rewards more steeply when they feel deprived than when they feel satiated $(t(119)=$ $2.26, p<.05)$. Within the low-sensitive-to-reward group, however, none of these contrasts is significant; see figure 5.

This pattern is obtained for each reward separately. That is, touching a bra leads to steeper delay discounting of money, cans of soda pop, and candy bars only among men with a sensitive reward system, but not after monetary satiation (see figs. 6, 7, and 8).

The effect of sexual cues on delay discounting is not likely to be driven by mood differences: no significant differences in mood, mood valence, and arousal were obtained between conditions, and adjusting for these variables as covariates in the reported analyses did not change the pattern of results, 
FIGURE 5

DELAY DISCOUNTING OF REWARDS

(STUDY 3)

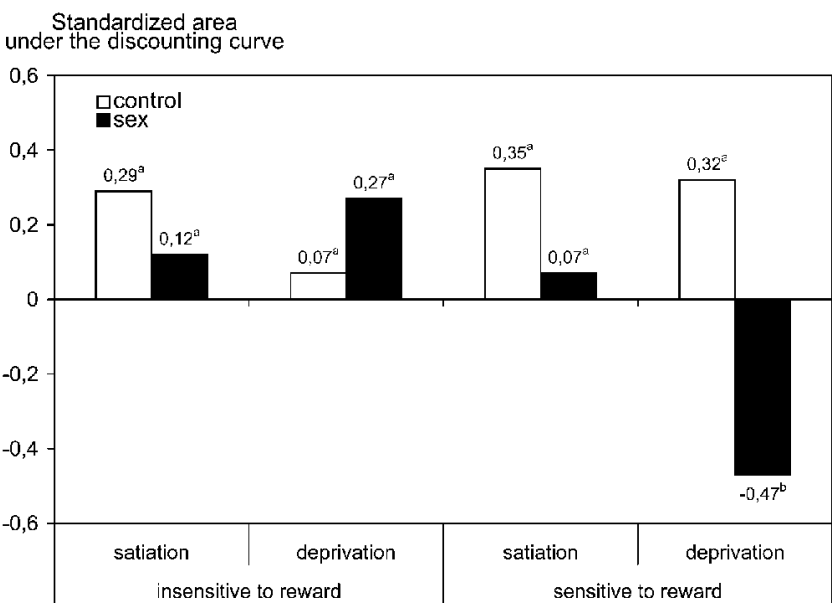

NOTE.-Different superscripts indicate that the difference between means is significant $(p \leq .05)$.

suggesting that mood did not mediate the effect of sexual cues on delay discounting.

\section{Discussion}

In this third study, we provided further support for the role of a general reward mechanism in delay discounting of rewards. We replicated the result of study 2 by demonstrating that the effect of sexual cues on delay discounting is moderated by BAS sensitivity. Furthermore, we demonstrated that the effect of sexual cues is not restricted to the monetary domain but generalizes to different types of reward, such as candy bars and cans of soda pop. This rules out an explanation in terms of mate attraction, because we obtained effects among rewards not directly related to material wealth. Finally, we found that satiating the induced motivational state dampens the appetitive response after exposure to "hot stimuli." Not only does this rule out an explanation in terms of behavioral effects of stereotype activation (Wheeler and Petty 2001) but these results are difficult to reconcile with a mere arousal account: it is unlikely that our satiation/deprivation manipulation would eliminate arousal induced by "hot stimuli" exposure.

\section{GENERAL DISCUSSION}

This research attempted to investigate whether exposure to "hot stimuli" leads to a general time perspective collapse toward the present. The current studies demonstrate that sexual appetite induces monetary craving. In line with neurological findings suggesting that rewards are processed similarly in the brain, we have provided evidence that a general reward system is giving rise to this effect. Among individuals with an insensitive reward system, sex cue exposure does not give rise to generalized impatience. Only when the reward system was sensitive enough to be activated by sex cue exposure was a time preference shift toward the present observed. We showed that the effect is not restricted to monetary rewards: sex cue exposure leads to impatience for candy bars and cans of soda pop as well. These results suggest that an induced sexual appetite instigates a greater urgency to consume anything rewarding. Further evidence for the role of the general reward system was provided by demonstrating that monetary satiation could attenuate the effect of sex cues on impatience in any domain. After touching a bra, monetary satiation leads to shallower discounting of candy bars and cans of soda pop than monetary deprivation. Additionally, we have ruled out explanations in terms of mood/arousal (Mick and Faure 1998; Rook and Gardner 1993; Tice et al. 2001), presence of individuals (Luo 2005), attractiveness of female models (Wilson and Daly 2004), cognitive load (Hinson et al. 2003; Shiv and Fedorikhin 1999), mate attraction (Roney 2003), and stereotype activation (Wheeler and Petty 2001).

Our brains include motivational mechanisms designed to ensure that we have sex when the situation is propitious for reproduction, eat when nutritionally deficient, drink when thirsty, and so forth. Prior research indicated that cues commonly associated with opportunities for having sex lead to an increase in motivation or desire to have sex. For instance, masturbating men report a greater willingness to take morally dubious measures to procure sex, find a much wider range of activities sexually appealing, and report a greater willingness to engage in risky sexual activities (Ariely and Loewenstein 2006). We demonstrated that this increase in appetitive motivation may emerge in domains that are unrelated to the cues that instigated the increase in appetitive

\section{FIGURE 6}

DELAY DISCOUNTING OF MONEY (STUDY 3)

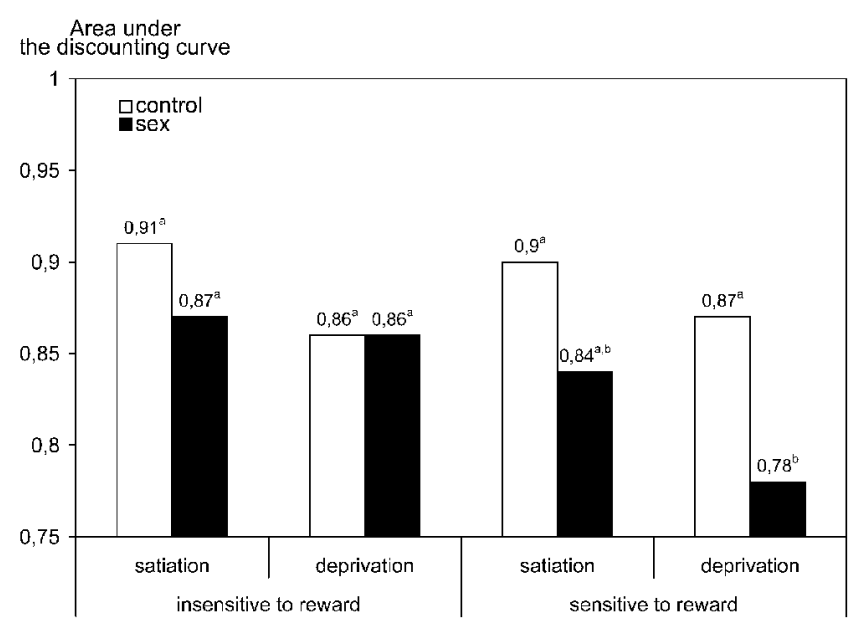

NOTE.-Different superscripts indicate that the difference between means is significant $(p \leq .05)$. 
FIGURE 7

DELAY DISCOUNTING OF CANS OF SODA POP (STUDY 3)

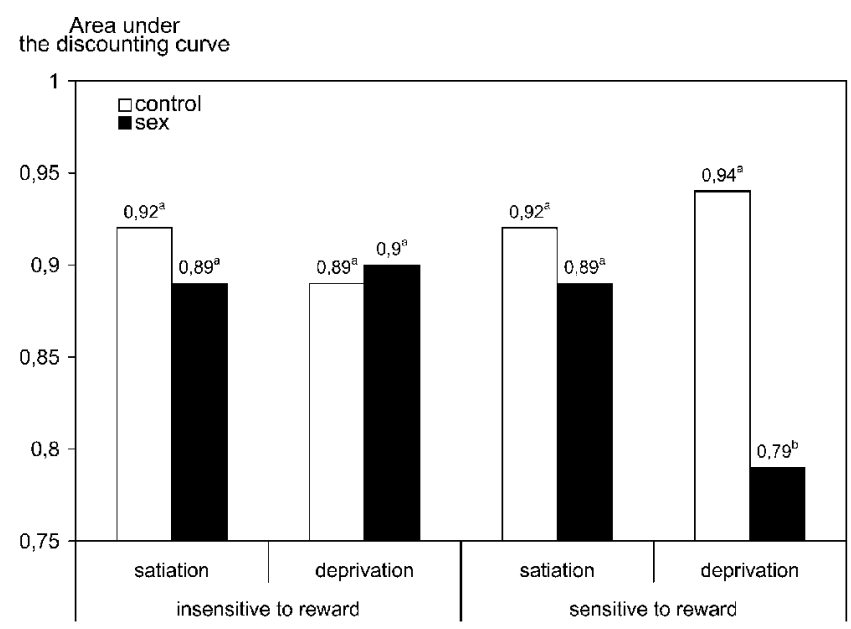

NoTE.-Different superscripts indicate that the difference between means is significant $(p \leq .05)$

motivation. Our research suggests that prior exposure to sexy stimuli may influence the choice between chocolate cake or fruit for dessert. As such, this series of experiments contributes to a growing body of research showing out-ofdomain effects of visceral states (Briers et al. 2006; Field et al. 2006; Giordano et al. 2002; Wilson and Daly 2004) and supports arguments for a common "neural currency" of reward (Montague and Berns 2002).

This is not to say that intertemporal choice would be regulated by one single valuation mechanism. Brain imaging research suggests that intertemporal choice can be viewed as a splice of two processes - an impulsive, affective, hot process and a more far-sighted, cognitive, cool process (Camerer et al. 2005; McClure et al. 2004). Different neurological regions are activated by decisions involving rewards available today and decisions involving intertemporal choices irrespective of delay. Parts of the affective system are preferentially activated by immediately available rewards. In contrast, more cognitive regions are engaged by intertemporal choices irrespective of delay (McClure et al. 2004). Thus, delay discounting reflects the operation of more than one single valuation mechanism (Montague et al. 2006). We would like to stress that our conclusions may be restricted to immediate rewards (i.e., short-run impatience is driven by the affective, limbic system; McClure et al. 2004) and may not generalize to choice between two delayed rewards. We think it is plausible that "hot stimuli" exposure may not lead to generalized impatience if individuals have to match a future reward (e.g., €15 in a year) with an even more delayed reward. Long-run patience is mediated by neurological activity within the more cognitive structures (McClure et al. 2004), which may be unaffected by "hot stimuli" exposure. Hence, this research provides evidence that one single mechanism may value all immediate rewards, but we remain ignorant about the specificity of the valuation mechanism for delayed rewards. Our conclusions may thus be restricted to decisions between now and the future.

It is appropriate to acknowledge the limitations of the current study. First, our samples were composed of young male students, which poses problems for generalization across populations. Indeed, prior research with female participants failed to find similar effects (Wilson and Daly 2004). Sex cues may not activate a general reward mechanism in women, but we nonetheless propose that "hot stimuli" exposure may lead to analogous effects within a female population. Indeed, Briers et al. (2006) found that an increase in desire for food, instigated by the scent of freshly baked brownies, leads to monetary craving among a female population. This brings us to the second limitation of the current research: we only addressed the effect of sexual cues. Nevertheless, we hypothesize that any increase in appetitive motivation may lead to generalized temporal myopia. For example, future research may explore the reverse effect of the current experiments: does monetary deprivation lead to steeper delay discounting of sexual rewards (e.g., specify the number of minutes of sexual activity with your favorite movie star you would require in 1 week to make you indifferent to receiving 15 minutes of sexual activity right now)? On the one hand, one may observe steeper discounting of sexual rewards after "hot stimuli" exposure and find similar results as reported above. On the other hand, individuals may derive more pleasure from delaying a sexual reward (Loewenstein 1987): a longer time delay may enhance the hedonic effect of anticipation of a sexual reward, which may lead to a shallower discounting function after "hot stimuli" exposure.

\section{FIGURE 8}

\section{DELAY DISCOUNTING OF CANDY BARS} (STUDY 3)

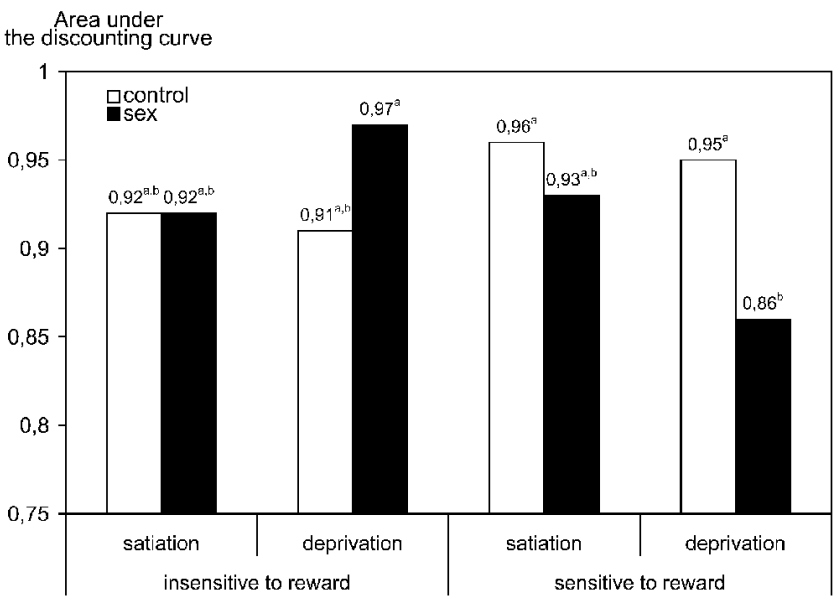

NoTE.-Different superscripts indicate that the difference between means is significant $(p \leq .05)$. 


\section{REFERENCES}

Aharon, Itzhak, Nancy Etcoff, Dan Ariely, Chris F. Chabris, Ethan O'Connor, and Hans C. Breiter (2001), "Beautiful Faces Have Variable Reward Value: fMRI and Behavioral Evidence," Neuron, 32 (3), 537-51.

Ariely, Dan and George Loewenstein (2006), "The Heat of the Moment: The Effect of Sexual Arousal on Sexual Decision Making," Journal of Behavioral Decision Making, 19 (2), 87-98.

Baddeley, Alan (1996), "Exploring the Central Executive," Quarterly Journal of Experimental Psychology, 49 (1), 5-28.

Baddeley, Alan, Dino Chincotta, and Anna Adlam (2001), "Working Memory and the Control of Action: Evidence from Task Switching," Journal of Experimental Psychology: General, 130 (4), 641-57.

Baumeister, Roy F. (2002), "Yielding to Temptation: Self-Control Failure, Impulsive Purchasing, and Consumer Behavior," Journal of Consumer Research, 28 (March), 670-76.

Blanton, Hart and Meg Gerrard (1997), "Effect of Sexual Motivation on the Perceived Riskiness of a Sexual Encounter: There Must Be Fifty Ways to Justify a Lover," Health Psychology, 16 (4), 374-79.

Briers, Barbara, Mario Pandelaere, Siegfried Dewitte, and Luk Warlop (2006), "Hungry for Money: The Desire for Caloric Resources Increases the Desire for Financial Resources and Vice Versa," Psychological Science, 17 (11), 939-43.

Camerer, Colin, George Loewenstein, and Drazen Prelec (2005), "Neuroeconomics: How Neuroscience Can Inform Economics," Journal of Economic Literature, 43 (1), 9-64.

Carver, Charles S. and Teri L.White (1994), "Behavioral Inhibition, Behavioral Activation, and Affective Responses to Impending Reward and Punishment: The BIS/BAS Scales," Journal of Personality and Social Psychology, 67 (2), 319-33.

Dholakia, Utpal M., Mahesh Gopinath, and Richard P. Bagozzi (2005), "The Role of Desires in Sequential Impulsive Choices," Organizational Behavior and Human Decision Processes, 98 (2), 179-94.

Eisenberger, Robert, Stephen Armeli, and Jean Pretz, (1998), "Can the Promise of Reward Increase Creativity?" Journal of Personality and Social Psychology, 74 (3), 704-14.

Estle, Sara J., Leonard Green, Joel Myerson, and Daniel D. Holt (2007), "Discounting of Monetary and Directly Consumable Rewards," Psychological Science, 18 (1), 58-63.

Field, Matt, Mary Santarcangelo, Harry Sumnall, Andrew Goudie, and Jon Cole (2006), "Delay Discounting and the Behavioural Economics of Cigarette Purchases in Smokers: The Effects of Nicotine Deprivation," Psychopharmacology, 186 (2), 255-63.

Förster, Jens, Nira Liberman, and E. Tory Higgins (2005), “Accessibility from Active and Fulfilled Goals," Journal of Experimental Social Psychology, 41 (3), 220-39.

Frederick, Shane, George Loewenstein, and Ted O'Donoghue (2002), "Time Discounting and Time Preference: A Critical Review," Journal of Economic Literature, 40 (2), 351-401.

Gilbert, Daniel T., Michael J. Gill, and Timothy D. Wilson (2002), "The Future Is Now: Temporal Correction in Affective Forecasting," Organizational Behavior and Human Decision Processes, 88 (1), 430-44.

Giordano, Louis A., Warren K. Bickel, George Loewenstein, Eric A. Jacobs, Lisa Marsch, and Gary J. Badger. (2002), "Opioid Deprivation Affects How Opioid-Dependent Outpatients Dis- count the Value of Delayed Heroin and Money," Psychopharmacology, 163 (2), 174-82.

Gray, Jeffrey A. (1987), "Perspectives on Anxiety and Impulsivity: A Commentary," Journal of Research in Personality, 21 (4), $493-509$.

(1990), "Brain Systems That Mediate Both Emotion and Cognition," Cognition and Emotion, 4 (3), 269-88.

Green, Leonard and Joel Myerson (2004): "A Discounting Framework for Choice with Delayed and Probabilistic Rewards," Psychological Bulletin, 130 (5), 769-92.

Griskevicius, Vladas, Robert B. Cialdini, and Douglas T. Kenrick, (2006), "Peacocks, Picasso, and Parental Investment: The Effects of Romantic Motives on Creativity," Journal of Personality and Social Psychology, 91 (1), 63-76.

Hinson, John M., Tina L. Jameson, and Paul Whitney, P. (2003), "Impulsive Decision Making and Working Memory," Journal of Experimental Psychology: Learning, Memory and Cognition, 29 (2), 298-306.

Hoch, Stephen J. and George Loewenstein (1991), "Time-Inconsistent Preferences and Consumer Self-Control," Journal of Consumer Research, 17 (March), 492-507.

Johnson, Matthew W. and Warren K. Bickel (2002), "Within-Subject Comparison of Real and Hypothetical Money Rewards in Delay Discounting," Journal of the Experimental Analysis of Behavior, 77 (2), 129-46.

Kirk, J. M. and A. W. Logue (1997), "Effects of Deprivation Level on Humans' Self-Control for Food Reinforcers," Appetite, 28 (3), 215-26.

Lagorio, Carla H. and Gregory J. Madden (2005), "Delay Discounting of Real and Hypothetical Rewards III: Steady-State Assessments, Forced-Choice Trials, and All Real Rewards," Behavioural Processes, 69 (2), 173-87.

Loewenstein, George (1987), "Anticipation and the Valuation of Delayed Consumption,” Economic Journal, 97 (387), 666-84.

_ (1996), "Out of Control: Visceral Influences on Behavior," Organizational Behavior and Human Decision Processes, 65 (3), 272-92.

Luo, Xueming (2005), "How Does Shopping with Others Influence Impulsive Purchasing?” Journal of Consumer Psychology, 15 (4), 288-94.

Madden, Gregory J., Andrea M. Begotka, Bethany R. Raiff, and Lana L. Kastern (2003), "Delay Discounting of Real and Hypothetical Rewards," Experimental and Clinical Psychopharmacology, 11 (2), 139-45.

Madden, Gregory J., Bethany R. Raiff, Carla H. Lagorio, Andrea M. Begotka, Angela M. Mueller, Daniel J. Hehli, and Ashley A. Wegener (2004), "Delay Discounting of Potentially Real and Hypothetical Rewards II: Between- and Within-Subject Comparisons," Experimental and Clinical Psychopharmacology, 12 (4), 251-61.

McClure, Samuel M., David I. Laibson, George Loewenstein, and Jonathan D. Cohen (2004), "Separate Neural Systems Value Immediate and Delayed Monetary Rewards," Science, 306 (5695), 503-7.

Mednick, Sarnoff A. (1962), "The Associative Basis of the Creative Process," Psychological Review, 69 (3), 220-32.

Metcalfe, Janet and Walter Mischel (1999), "A Hot/Cool-System Analysis of Delay of Gratification: Dynamics of Willpower," Psychological Review, 106 (1), 3-19.

Mick, David Glen and Corinne Faure (1998), "Consumer SelfGifts in Achievement Contexts: The Role of Outcomes, Attributions, Emotions, and Deservingness," International Journal of Research in Marketing, 15 (July), 293-307. 
Mischel, Walter and Ebbe B. Ebbesen (1970), “Attention in Delay of Gratification," Journal of Personality and Social Psychology, $16(2), 329-37$.

Mitchell, Suzanne H. (2004), "Effects of Short-Term Nicotine Deprivation on Decision-Making: Delay, Uncertainty, and Effort Discounting," Nicotine and Tobacco Research, 6 (5), 819-28.

Montague, P. Read and Gregory S. Berns (2002), "Neural Economics and the Biological Substrates of Valuation," Neuron, 36 (2), 265-84.

Montague, P. Read, Brooks King-Casas, and Jonathan D. Cohen (2006), "Imaging Valuation Models in Human Choice," Annual Review of Neuroscience, 29 (July), 417-48.

Morris, Jon D. (1995), “Observations: SAM: The Self-Assessment Manikin - an Efficient Cross-cultural Measurement of Emotional Response," Journal of Advertising Research, 35 (6), 63-68.

Myerson, Joel, Leonard Green, and Warusawitharana, M. (2001), "Area under the Curve as a Measure of Discounting," Journal of the Experimental Analysis of Behavior, 76 (2), 235-43.

Nelson, Leif D. and Evan L. Morrison (2005), "The Symptoms of Resource Scarcity: Judgments of Food and Finances Influence Preferences for Potential Partners," Psychological Science, 16 (2), 167-73.

Nisbett, Richard E. and David E. Kanouse (1969), "Obesity, Food Deprivation, and Supermarket Shopping Behavior," Journal of Personality and Social Psychology, 12 (4), 289-94.

Oppenheimer, Daniel M., Tom Meyvis, and Nicolas Davidenko (2007), "Instructional Manipulation Checks: Detecting Satisficing to Increase Statistical Power in Survey Studies," working paper, Social Science Research Network. http://www .ssrn.com/abstract $=1003424$.

Ramanathan, Suresh and Geeta Menon (2006), "Time-Varying Effects of Chronic Hedonic Goals on Impulsive Behavior," Journal of Marketing Research, 43 (4), 628-41.

Read, Daniel and Barbara van Leeuwen (1998), "Predicting Hunger: The Effects of Appetite and Delay on Choice," Organizational Behavior and Human Decision Processes, 76 (2), 189-205.

Roney, James R. (2003), "Effects of Visual Exposure to the Opposite Sex: Cognitive Aspects of Mate Attraction in Human Males," Personality and Social Psychology Bulletin, 29 (3), 393-404.

Rook, Dennis W. and Meryl P. Gardner (1993), "In The Mood: Impulse Buying's Affective Antecedents," in Research in Consumer Behavior, Vol. 6, ed. Janeen Arnold-Costa and Russell Belk, Greenwich, CT: JAI, 1-28.

Schwarz, Norbert (1999), "Self Reports: How the Questions Shape the Answers," American Psychologist, 54 (2), 93-105.

Shiv, Baba and Alexander Fedorikhin (1999), "Heart and Mind in
Conflict: The Interplay of Affect and Cognition in Consumer Decision Making," Journal of Consumer Research, 26 (December), 278-92.

Soman, Dilip, George Ainslie, Shane Frederick, Xiuping Li, John Lynch, Page Moreau, Andrew Mitchell, Daniel Read, Alan Sawyer, Yaacov Trope, and Klaus Wertenbroch (2005), "The Psychology of Intertemporal Discounting: Why Are Distant Events Valued Differently from Proximal Ones?" Marketing Letters, 16 (3-4), 347-60.

Stark, Rudolf, Anne Schienle, Cornelia Girod, Bertram Walter, Peter Kirsch, Carlo Blecker, Ullrich Ott, Axel Schäfer, Gebhard Sammer, Mark Zimmermann, and Dieter Vaitl (2005), "Erotic and Disgust-Inducing Pictures: Differences in the Hemodynamic Responses of the Brain," Biological Psychology, 70 (1), 19-29.

Thaler, Richard H. (1981), "Some Empirical Evidence on Dynamic Inconsistency," Economic Letters, 8 (3), 201-7.

Tice, Diane M., Ellen Bratslavsky, and Roy F. Baumeister (2001), "Emotional Distress Regulation Takes Precedence over Impulse Control: If You Feel Bad, Do It!” Journal of Personality and Social Psychology, 80 (1), 53-67.

Toms, Margaret, Neil Morris, and Deborah Ward (1993), "Working Memory and Conditional Reasoning," Quarterly Journal of Experimental Psychology, 46 (4), 679-99.

Torrubia, Rafael, César Avila, Javier Moltó, and Xavier Caseras (2001), "The Sensitivity to Punishment and Sensitivity to Reward Questionnaire (SPSRQ) as a Measure of Gray's Anxiety and Impulsivity Dimensions," Personality and Individual Differences, 31 (6), 837-62.

Tukey, John W. (1977), Exploratory Data Analysis, Reading, MA: Addison-Wesley.

Van den Bergh, Bram and Siegfried Dewitte (2006), "Digit Ratio (2D:4D) Moderates the Impact of Sexual Cues on Men's Decisions in Ultimatum Games," Proceedings of the Royal Society B: Biological Sciences, 273 (1597), 2091-95.

Wadhwa, Monica, Baba Shiv, and Stephen M. Nowlis (2006), "A Bite to Whet the Reward Appetite: The Effect of Sampling on Reward-Seeking Behaviors," working paper, Graduate School of Business, Stanford University.

Watson, David, Lee Anna Clark, and Auke Tellegen (1988), "Development and Validation of Brief Measures of Positive and Negative Affect: The PANAS Scales," Journal of Personality and Social Psychology, 54 (6), 1063-70.

Wheeler, S. Christian and Richard E. Petty (2001), "The Effects of Stereotype Activation on Behavior: A Review of Possible Mechanisms," Psychological Bulletin, 127 (6), 797-826.

Wilson, Margo and Martin Daly (2004), "Do Pretty Women Inspire Men to Discount the Future?" Proceedings of the Royal Society London B, 271 (No. 4, supplement), S177-S179. 